\title{
The Rhesus Macaque as a Translational Model for Neurodegeneration and Alzheimer's Disease
}

\author{
Gail A. Stonebarger ${ }^{1,2}$, Heather A. Bimonte-Nelson ${ }^{3,4}$ and Henryk F. Urbanski ${ }^{1,2 *}$ \\ ${ }^{1}$ Division of Neuroscience, Oregon National Primate Research Center, Beaverton, OR, United States, ${ }^{2}$ Department \\ of Behavioral Neuroscience, Oregon Health and Science University, Portland, OR, United States, ${ }^{3}$ Department \\ of Psychology, Arizona State University, Tempe, AZ, United States, ${ }^{4}$ Arizona Alzheimer's Consortium, Phoenix, AZ, \\ United States
}

A major obstacle to progress in understanding the etiology of normative and pathological human brain aging is the availability of suitable animal models for experimentation. The present article will highlight our current knowledge regarding human brain aging and neurodegeneration, specifically in the context of Alzheimer's disease (AD). Additionally, it will examine the use of the rhesus macaque monkey as a pragmatic translational animal model in which to study underlying causal mechanisms. Specifically, the discussion will focus on behavioral and protein-level brain changes that occur within the central nervous

OPEN ACCESS

Edited by:

Changning Wang, Harvard Medical School,

United States

Reviewed by:

Qihan Li,

Chinese Academy of Medical Sciences and Peking Union Medical

College, China

Dongdong Qin,

Yunnan University of Traditional

Chinese Medicine, China

*Correspondence: Henryk F. Urbansk urbanski@ohsu.edu

Received: 30 June 2021 Accepted: 12 August 2021 Published: 03 September 2021

Citation:

Stonebarger GA,

Bimonte-Nelson HA and Urbanski HF (2021) The Rhesus Macaque as a Translational Model for Neurodegeneration

and Alzheimer's Disease.

Front. Aging Neurosci. 13:734173.

doi: 10.3389/fnagi.2021.734173 system (CNS) of aged monkeys, and compare them to the changes observed in humans during clinically normative aging and in AD.

Keywords: amyloid beta, animal models, brain aging, clinical aging, cognitive decline, non-human primate, phosphorylated tau

\section{INTRODUCTION}

\section{Alzheimer's Disease}

By the year 2050, nearly one in four people in the United States is expected to be 65 years of age or older, and people over 65 are predicted to make up $61.9 \%$ of the global population (U.S. Census Bureau, 2020). This change in population dynamics is likely to have a major impact on the health care systems. For instance, even in the absence of overt neurodegeneration, older adults show gradual changes in mental processes such as attention, memory retrieval, processing speed, and executive function (Harada et al., 2013; Schott, 2017). However, half of adults in the United States aged 50-64 years consider themselves at least somewhat at risk for developing overt dementia such as Alzheimer's disease (AD), and $37 \%$ report having a family member with dementia (Maust et al., 2020). These concerns encompass the wide range of $\mathrm{AD}$ symptoms-including progressive memory loss, agitation, language deficits, depression, mood disturbances, and even psychosis-and are not unfounded, as a growing percentage of the population does develop AD (Schott, 2017; Mehla et al., 2020). The most common symptoms of $\mathrm{AD}$ are the severe dementia and neuropsychiatric complaints involved in advanced $\mathrm{AD}$, with $60-70 \%$ of dementia cases being attributable to $\mathrm{AD}$ (Dey et al., 2017).

\section{Pathological Hallmarks of AD in Humans}

Many physical changes in the brain have been identified across clinically normative aging, such as mild brain atrophy, gliosis, and accumulation of intracellular and extracellular proteins 
(Marner et al., 2003; Freeman et al., 2008; Schott, 2017; Furcila et al., 2018). These changes do appear to alter cognitive function, even in healthy aging humans (Furcila et al., 2018). However, patients with $\mathrm{AD}$ show these symptoms at a younger age, which sets $\mathrm{AD}$ patients onto a separate, pathological, brain aging trajectory. AD patients additionally show overt neuron loss, which is not present in normative aging (Freeman et al., 2008). Accordingly, they also reach higher levels of pathology than their non-AD peers (Duyckaerts et al., 2009). Two hallmarks of AD pathology are amyloid beta $(A \beta)$ : A protein which aggregates into extracellular plaques and is formed from cleavage of the APP, and phosphorylated tau ( $\mathrm{p}$-tau): A microtubule-associated protein which becomes hyperphosphorylated and accumulates intracellularly (Duyckaerts et al., 2009; see Figure 1 for protein aggregation of each). Notably, these proteins are present in the non-AD aging brain as well; however, specific protein modifications are necessary for the clinical diagnosis of $\mathrm{AD}$ (Furcila et al., 2018).

\section{Current Pharmacological Therapies}

There has been very limited efficacy in preventing the onset of some new memory-related symptoms with memantine, an N-methyl-D-aspartate (NMDA) receptor antagonist, cholinesterase inhibiters such as donepezil, and a recently approved combination of the two classes (Miziak et al., 2021). However, there are no current interventions which treat the etiology of or even prevent the progression of $\mathrm{AD}$, in spite of many studies and clinical trials (i.e., Farlow et al., 2012; Ostrowitzki et al., 2017; Cummings et al., 2018; Egan et al., 2019; Haass and Levin, 2019). Most recently, aducanumab, an antibody shown to reduce amyloid beta accumulation, was FDA-approved using a fast-tracked pathway (U.S. Food \& Drug Administration, 2021). This approval, however, was based solely on the surrogate endpoint of plaque reduction, so the therapeutic potential of aducanumab is unknown especially in the context of symptomatic relief. This is because $A \beta$ plaques do not reliably correlate with cognitive decline, and this and previous amyloidlowering drugs have failed to consistently slow dementia onset (Nelson et al., 2012; Sevigny et al., 2016). Therefore, to make significant advances in our understanding of the pathways and neurodegenerative trajectories that underlie $\mathrm{AD}$, and to develop safe and effective therapies, it is important to develop and characterize appropriate experimental animal models of AD. These preclinical models inform and drive novel investigations in the clinical realm, underscoring the importance of promoting cross-talk between preclinical and clinical science, as well as the critical nature of translational science for therapeutics.

\section{Preclinical Rodent Models of AD}

Rodents are the most commonly used laboratory animals and have relatively short lifespans to allow for rapid experimental turnaround in aging studies. They do not, however, naturally develop measurable pathological features of $\mathrm{AD}$ such as $\mathrm{A} \beta$ plaques or p-tau tangles (LaFerla and Green, 2012). Several methods have been employed to mitigate this lack of overt pathology, generally via modifications to genes which are known risk factors for $\mathrm{AD}$, such as amyloid precursor protein (APP), apolipoprotein E, and presenilin (Webster et al., 2014). Other models involve adeno-associated virus (AAV) vectors and floxed genes (i.e., temporally controlled and mediated genes), induction of pathology via direct or indirect addition of $A \beta$ and/or $p$-tau, or sometimes direct insertion of clinical genes or proteins found in $\mathrm{AD}$ patients. For examples of these manipulations, see Alzforum (2021). These models allow great flexibility and temporal control over induction of $\mathrm{AD}$ characteristics which cannot be achieved with sporadically occurring clinical AD. Specific realms of memory can be probed on a basic level using rodent behavioral paradigms; for instance: set-shifting, spatial navigation, working memory, perseveration, and many other typically affected forms of cognition are measurable in rodents. These paradigms provide valuable insights into specific realms of memory as well as molecular mechanisms of aging. Conversely, some complex cognitive measures-such as the neurocognitive testing considered to be the clinical gold standard-are impractical or sometimes even impossible to use in characterizations of AD-like symptomology in rodents (Harada et al., 2013; Webster et al., 2014; Deb et al., 2017; Ingram and De Cabo, 2017; Colman, 2018). Therefore, collectively, when assessing clinical hallmarks of $\mathrm{AD}$, the rodent model provides a basis for informing studies in higher mammals.

Mice and rats are short-lived, allowing for study of the aging process in a relatively contracted time frame, but this abbreviated lifespan also limits chronological aging. For instance, 2 years (the average lifespan of a laboratory mouse; Flurkey et al., 2007) simply may not be enough time for pathological proteins such as $\mathrm{A} \beta$ or $\mathrm{p}$-tau to accumulate and have neurodegenerative effects. As seen in the interactive visualization by Alzforum (2021), many mouse models do recapitulate aspects of $\mathrm{AD}$ - for example, APP processing (Figures 1A-C) via genetic induction-and they have been crucial to our understanding of mechanisms of aging and $\mathrm{AD}$. To gain a holistic understanding of the disease, a more robust, naturally-occurring model is optimal. This model must also be highly clinically translational in order to probe complex cognitive functioning, temporal dynamics, and noncognitive neuropsychiatric symptoms. These shortcomings in rodent models may be complemented with the study of the rhesus macaque to model $\mathrm{AD}$ and aging. Taken together, the use of small laboratory rodents is paramount for studying cellular and molecular aspects of $\mathrm{AD}$ pathology, but a more complete clinically translational model is needed to recapitulate subtle behavioral symptoms and sporadic onset of both AD and normative aging mechanisms.

\section{THE RHESUS MACAQUE MODEL OF NORMATIVE AND PATHOLOGICAL BRAIN AGING}

The rhesus macaque (Macaca mulatta) shows close genetic homology to humans (Rhesus Macaque Genome Sequencing Analysis Consortium, Gibbs et al., 2007), has a greatly extended lifespan relative to rodents (Mattison et al., 2017; Stonebarger et al., 2020), is capable of performing complex and prefrontal cortex (PFC)-dependent cognitive tests similar to those used in 


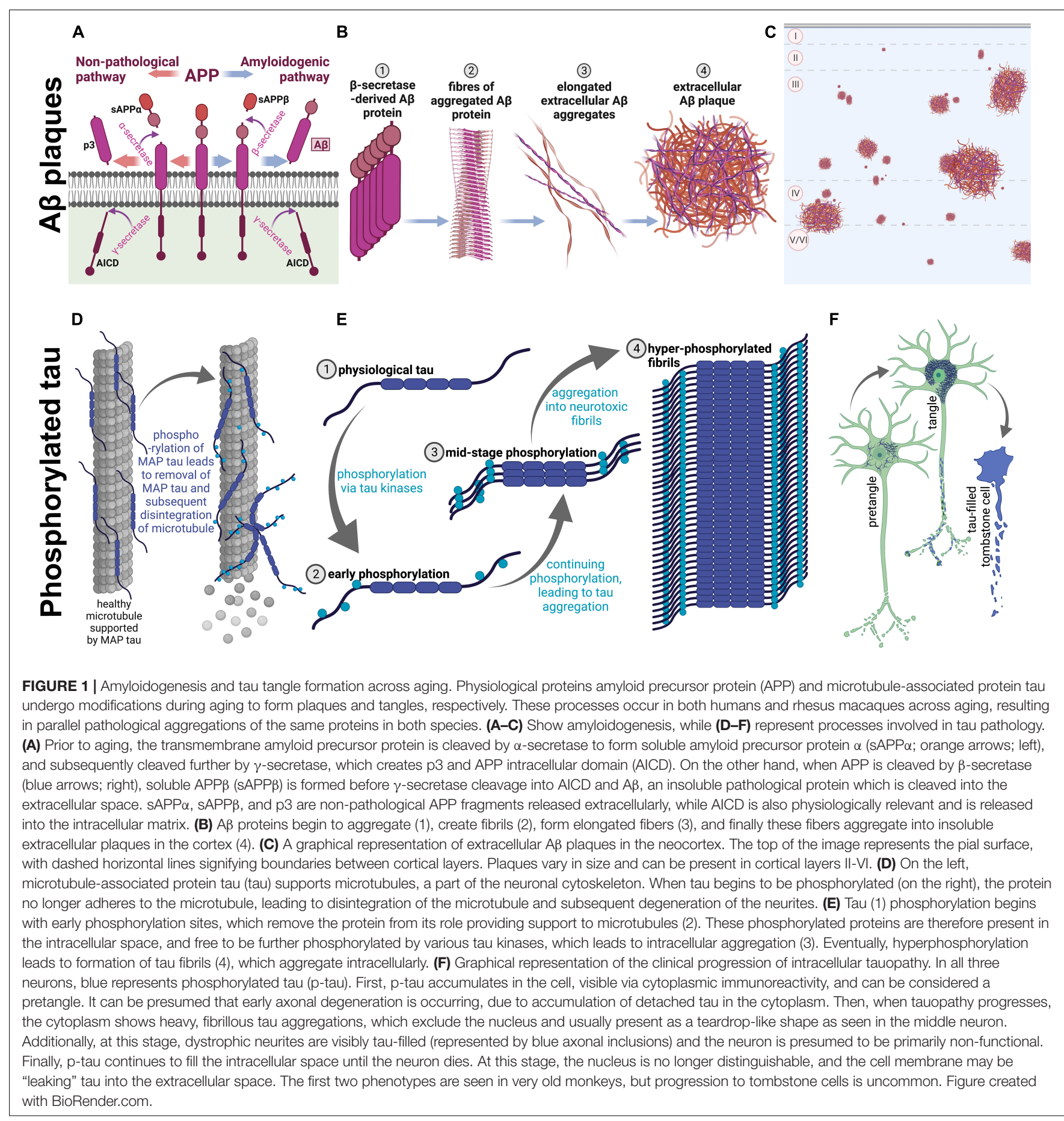

the clinic (Rapp, 1989; Peters et al., 1996; Moore et al., 2006; Nagahara et al., 2010) and also shows similar life stages; i.e., a prolonged childhood, adolescence, adulthood, and cognitive and hormonal aging declines (Downs and Urbanski, 2006; Messaoudi et al., 2011; Sorwell et al., 2012; Mattison et al., 2017). For these reasons, the rhesus macaque may represent a more holistic and systems approach to studying mechanisms underlying human aging. Additionally, it has been commonly known for decades that rhesus macaques develop cortical and subcortical A $\beta$ plaques-which represents the natural formation and aggregation of this protein-and there is evidence that this involves the same pathways as in human brain aging (Heilbroner and Kemper, 1990; Uno, 1993; Stonebarger et al., 2020).

\section{Cognitive Decline}

Because rhesus macaques are capable of more complex learning and memory tasks and have longer lifespans compared to rodents, they represent a valuable translational animal 
model with which to study longitudinal changes in cognitive function. For instance, many behavioral batteries include neuropsychological tests that closely resemble those used to clinically assess cognitive decline; i.e., monkeys are capable of performing testing with the same complexity and nearly identical parameters as multiple tests used in the clinic. To this end, many researchers have observed a gradual but significant decline in cognitive ability which occurs over the course of macaque late adulthood (Lai et al., 1995; Herndon et al., 1997; Moore et al., 2003, 2006). For example, short-term memory, taskdependent memory, two-choice discriminations, abstraction, and set-shifting have all been shown to be impaired in old rhesus macaques compared to young, or even in some instances also compared to middle-aged monkeys (Bartus et al., 1978; Rapp and Amaral, 1989; Lai et al., 1995; Herndon et al., 1997; Voytko, 1999; Moore et al., 2003). Importantly, as in humans, this decline is progressive such that "early aged" monkeys (1923 years), "middle-aged" monkeys (24-29 years), and "oldest-old" $(30+$ years) show different levels of cognitive performance. Each age group shows impairments compared to the group directly younger in delayed non-match to sample tasks and non-spatial delayed recognition span test (Herndon et al., 1997; Harada et al., 2013). Like humans, variability in rhesus cognitive scores also increases with age (Schott, 2017). However, even considering deficits seen in animals with the lowest cognitive scores, aging macaques do not show decline of cognitive function as rapidly or to such severe impairment as humans with AD (Hara et al., 2012). After decades of cognitive aging research in the rhesus macaque, the primary takeaway points seem to be that the rhesus macaque does not model overt dementia such as $\mathrm{AD}$, but instead models the natural decline in various cognitive domains seen in healthy aging humans. It remains to be seen whether pathological tauopathy is correlated with cognitive decline in the rhesus macaque, as amyloid plaques are not consistently predictive of cognitive decline in monkeys (Emborg, 2017).

\section{Amyloid Beta}

For decades, the progression and accumulation of $A \beta$ extracellular plaques in the rhesus macaque has been welldocumented. For example, in 1993 Uno reported amyloid plaques in $40-66 \%$ of rhesus macaques aged $20-30$ years (Uno, 1993), and as detection methods have improved in the last three decades, it may be that an even higher percentage of aged animals show amyloidosis. However, at least in the PFC, amyloidosis levels do not reach the threshold for clinical AD. For instance, brains of AD patients tend to show 10-20\% area coverage of plaques in the PFC (O'Brien and Wong, 2011; Smith et al., 2019). Furthermore, it was recently shown that even the oldest known rhesus macaques in the world do not experience amyloid levels naturally higher than 5-6\% coverage, depending on the region of interest (Stonebarger et al., 2020). These PFC data could indicate that globally, $\mathrm{A} \beta$ simply does not accumulate enough in the rhesus brain to be overtly neurotoxic. Nevertheless, a majority of studies using various methodologies have confirmed an increase in rhesus cerebral amyloid across age, which parallels findings in the clinic (Heilbroner and Kemper, 1990; Uno, 1993; Stonebarger et al., 2020). Moreover, building on this observation, there have been successful attempts to inducerather than replicate-a phenotype that more closely parallels the human $\mathrm{AD}$ brain environment in multiple species of macaques (Forny-Germano et al., 2014; Beckman et al., 2019). Altogether, these $A \beta$ plaque data strongly suggest that the same aging processes occur in both human and rhesus macaques, but that they may be less developed in the rhesus brain.

\section{Phosphorylated Tau}

Until recently, the rhesus macaque had been accepted as an incomplete model of $\mathrm{AD}$, or potentially even dismissed fully as a model of natural clinical aging, due to the lack of neuronal p-tau detection. Indeed, for decades, rhesus macaques were considered not to develop p-tau naturally. This has presented a challenge in phylogenetically synthesizing the evolution of p-tau tanglesas chimpanzees, baboons, gorillas, and cynomolgus monkeys have all shown evidence of cortical tauopathy (Schultz et al., 2000; Oikawa et al., 2010; Perez et al., 2013; Edler et al., 2020), along with some smaller simians such as marmosets and lemurs (Giannakopoulos et al., 1997; Sharma et al., 2019). Rhesus monkeys, a key evolutionary link, have not historically shown immunoreactivity to p-tau (Peters et al., 1996; Zhang et al., 2019). However, in 2018 Paspalas et al. reported detection of p-tau in the rhesus macaque entorhinal cortex, which has led to a resurgence in interest surrounding non-human primate $\mathrm{p}$-tau. In addition to this initial landmark study, laboratories are currently researching both natural p-tau accumulation and tauopathy induction models in the rhesus macaque (i.e., Beckman et al., 2021; Datta et al., 2021). There is still much work to be done characterizing natural tauopathy in the rhesus macaque, but it has generally been observed that most monkeys do not live long enough to develop pathological tauopathy. For instance, most overt tauopathy appears to occur mainly in macaques over the age of 30 years, and very few primate facilities have a cohort of these very old animals (Paspalas et al., 2018; Datta et al., 2021). Therefore, non-human primate induction models become highly relevant: if the same natural pathways that lead to tauopathy can be dysregulated or stimulated earlier in life, researchers can study the etiology and progression of the proteinopathy across many years, and monkeys would likely achieve more advanced states of tauopathy. This could mimic neurodegeneration or advanced stages of human brain aging, which can inform clinical progression of the disease; if researchers can induce tauopathy into a further progressed state in the macaque, the inverse processes are logical targets for early AD intervention. It should be emphasized that relatively few rhesus monkeys live beyond $\sim 25$ years, which is prior to the age at which they begin to show significant hippocampal tauopathy (Paspalas et al., 2018; Datta et al., 2021); thus, the systematic study of p-tau induction will be critically important in further developing the macaque $\mathrm{AD}$ model.

Taken together, the recent $\mathrm{p}$-tau data question the traditional dogma that non-human primates do not develop $\mathrm{AD}$ due to a lack of p-tau accumulation. The primary physical AD hallmarks: clinically relevant $\mathrm{p}$-tau intra-neuronal tangles and $\mathrm{A} \beta$ plaques, have now been observed in monkeys; so why don't they show a full cognitive $\mathrm{AD}$ phenotype? 


\section{Neuronal Death}

Mid- and late- stage clinical AD result in significant neuronal death in subcortical structures such as the hippocampus, and in the neocortex (Furcila et al., 2018). To fully capitulate an AD phenotype, the rhesus macaque would need to show significant cell loss in areas that are sensitive to aging. Thus far, there is only one report of neuronal loss in the rhesus macaque with loss indicated in PFC Brodmann Area 8a (Smith et al., 2004). However, data from Stonebarger et al. (2020) included a larger cohort of monkeys across more of the lifespan, and this expanded cohort did not show age-related neuronal loss in the PFC. Similarly, no neuronal loss was seen across multiple rhesus macaque brain regions, including the PFC, hypothalamus, and visual cortex (Peters et al., 1994, 1998; Roberts et al., 2012; Giannaris and Rosene, 2012; Stonebarger et al., 2020). Notably, the hippocampus has not to our knowledge been stereologically analyzed across age in the rhesus macaque. This brain region is highly sensitive to cognitive and pathological aging changes, critical to memory consolidation, and undergoes significant shrinkage in AD (Freeman et al., 2008; Squire et al., 2015). While the lack of neuron loss in all other analyzed areas seems to indicate that rhesus macaques do not naturally lose neurons as they age, it is interesting to note that the multiple cohorts of rhesus monkeys have shown p-tau tangles in the hippocampus (i.e., Paspalas et al., 2018; Datta et al., 2021). Furthermore, in clinical studies it has been shown that p-tau tangles are associated with cell death-one of the primary drivers of cognitive decline in AD (Peters et al., 1994; Datta et al., 2021). Taken together, the overall consensus is that rhesus macaques do not show the distinctive neuronal loss that characterizes the later stages of $\mathrm{AD}$ in humans, and which is associated with dementia.

\section{CONCLUSION AND FUTURE PERSPECTIVES}

Clinical studies can guide future directions of rodent behavioral and molecular research with regard to normal and pathological aging, which in turn can inform non-human primate research thereby potentially impacting clinical outcomes. This crossspecies integrative approach is impractical, however, without detailed characterization of aging and AD-like symptomology in the most common preclinical non-human primate: the rhesus macaque. Importantly, in the rhesus macaque model of aging, all four major hallmarks of $\mathrm{AD}$-significant cognitive decline, amyloid beta plaques, p-tau tangles, and neuronal death-do not reach the pathological levels of clinical $\mathrm{AD}$. Cognitive decline in aged rhesus monkeys parallels the healthy aging human very well, in that gradual, incremental decline is seen across age, beginning in middle age or later, depending on the task. Amyloid beta plaques do naturally accumulate in the rhesus macaque brain, especially in sensitive areas such as the PFC. Importantly, however, the area coverage of these plaques in rhesus monkey brains is not high enough to be considered an $\mathrm{AD}$ phenotype relative to diagnostic hallmarks in the clinic. While the recent discovery of naturally occurring p-tau in the

\section{Clinical aging

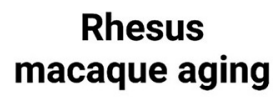

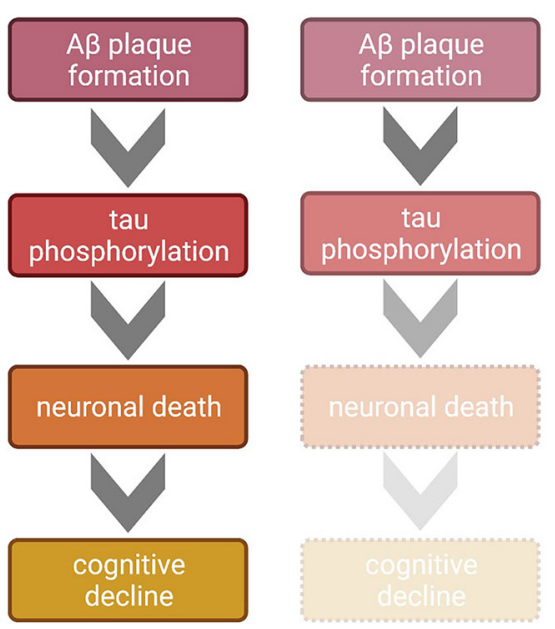

FIGURE 2 | Comparison of clinical and non-human primate progression of brain aging according to the generally accepted amyloid cascade hypothesis of $A D$. Clinically, $A \beta$ aggregates and triggers hyperphosphorylation of tau. This tauopathy accumulates intracellularly to lead to neuronal death, which is a primarily proposed mechanism of cognitive decline. Mid to late stages of AD result in the full clinical cascade visualized on the left. However, even in the oldest known rhesus macaques, this trajectory is incomplete; $A \beta$ plaques and tauopathy do not reach levels seen in the clinical AD cases. Therefore, because tauopathy only reaches early stages of phosphorylation, cell death and the subsequent sharp cognitive decline are absent in the monkey model, which renders the rhesus macaque more suitable for naturally modeling normative clinical brain aging or early stages of AD. Figure created with BioRender.com.

rhesus monkey brain is indeed exciting, data thus far indicate that the amount of p-tau detected is not in line with the classically accepted progression and advanced conditions of neurofibrillary tangles that represent hallmarks of AD. However, four distinct patterns of tau progression have recently been proposed; therefore, it is unclear which of these trajectories monkey p-tau most closely resembles (Vogel et al., 2021). Finally, neuronal death is an established AD phenotype, but this hallmark has not been obvious even in the very oldest rhesus macaques in studies to date. Taken altogether, it is evident that the rhesus macaque does not develop overt $\mathrm{AD}$, even though the CNS conditions represent subclinical variations of $\mathrm{AD}$ hallmarks, as visualized in Figure 2. Notably, this environment can be experimentally leveraged in a systematic fashion, setting the stage for induction or mitigation models in which various methods can be employed to exacerbate or attenuate existing pathology (i.e., Beckman et al., 2021).

Due to these temporal restrictions along with the very recent discovery of naturally arising p-tau in the rhesus macaque, true non-human primate models of $\mathrm{AD}$ are severely lacking. Monkeys, like humans, show great variability in phenotypical aging, meaning even in aged animals these hallmarks are not present in the entire population. This makes a natural aging macaque an expensive and unreliable model for $\mathrm{AD}$. This 
highlights the need for inducible non-human primate models of neurodegeneration. Notably, this environment has begun to be experimentally leveraged in a systematic fashion, setting the stage for induction or mitigation models in which various methods can be employed to exacerbate or attenuate existing pathology (i.e., Beckman et al., 2021). For instance, both rhesus and cynomologus macaques-a close genetic relativehave been shown to develop p-tau tangles in response to the injection of $\mathrm{A} \beta$ oligomers; however, even when induced tangles aggregate earlier in the lifespan, there is no recorded cell death (Lyra e Silva et al., 2019). Importantly, these are early experimental models, and work continues to be done to both characterize and exacerbate brain pathology in the aging monkey. Therefore, the rhesus macaque, while not an ideal natural model of clinical $\mathrm{AD}$, has the potential to be an excellent resource with which to study factors that contribute to the etiology, exacerbation, and root causes of the

\section{REFERENCES}

Alzforum (2021). Research Models: Alzheimer's Disease. Available online at: https://www.alzforum.org/research-models/alzheimers-disease (accessed June $8,2021)$.

Bartus, R. T., Fleming, D., and Johnson, H. R. (1978). Aging in the rhesus monkey: debilitating effects on short-term memory. J. Gerontol. 33, 858-871. doi: 10 . 1093/geronj/33.6.858

Beckman, D., Chakrabarty, P., Ott, S., Dao, A., Zhou, E., Janssen, W. G., et al. (2021). A novel tau-based rhesus monkey model of Alzheimer's pathogenesis. Alzheimers Dement. 17, 933-945. doi: 10.1002/alz. 12318

Beckman, D., Ott, S., Donis-Cox, K., Janssen, W. G., Bliss-Moreau, E., Rudebeck, P. H., et al. (2019). Oligomeric A? in the monkey brain impacts synaptic integrity and induces accelerated cortical aging. Proc. Natl. Acad. Sci. U.S.A., 116, 26239-26246. doi: 10.1073/pnas.1902301116

Colman, R. J. (2018). Non-human primates as a model for aging. Biochem. Biophys. Acta Mol. Basis Dis. 1864, 2733-2741. doi: 10.1016/j.bbadis.2017. 07.008

Cummings, J. L., Cohen, S., van Dyck, C. H., Brody, M., Curtis, C., Cho, W., et al. (2018). ABBY: a phase 2 randomized trial of crenezumab in mild to moderate Alzheimer disease. Neurology 90, E1889-E1897. doi: 10.1212/WNL. 0000000000005550

Datta, D., Leslie, S. N., Wang, M., Morozov, Y. M., Yang, S., Mentone, S., et al. (2021). Age-related calcium dysregulation linked with tau pathology and impaired cognition in non-human primates. Alzheimers Dement. 17, 920-932. doi: $10.1002 /$ alz. 12325

Deb, A., Thornton, J. D., Sambamoorthi, U., and Innes, K. (2017). Direct and indirect cost of managing Alzheimer's disease and related dementias in the United States. Expert Rev. Pharmacoecon. Outcomes Res. 2, 189-202. doi: 10. 1080/14737167.2017.1313118

Dey, A., Bhattacharya, R., Mukherjee, A., and Pandey, D. K. (2017). Natural products against Alzheimer's disease: pharmaco-therapeutics and biotechnological interventions. Biotechnol. Adv. 35, 178-216. doi: 10.1016/j.biotechadv.2016.12.005

Downs, J. L., and Urbanski, H. F. (2006). Neuroendocrine changes in the aging reproductive axis of female rhesus macaques (Macaca mulatta). Biol. Reprod. 75, 539-546. doi: 10.1095/biolreprod.106.051839

Duyckaerts, C., Delatour, B., and Potier, M. C. (2009). Classification and basic pathology of Alzheimer disease. Acta Neuropathol. 118, 5-36. doi: 10.1007/ s00401-009-0532-1

Edler, M. K., Munger, E. L., Meindl, R. S., Hopkins, W. D., Ely, J. J., Erwin, J. M., et al. (2020). Neuron loss associated with age but not Alzheimer's disease discussed mechanisms, including genetics, hormone milieu, diet, trauma, and infection.

\section{AUTHOR CONTRIBUTIONS}

GS drafted the current manuscript, with writing and editing contributions from HB-N and HU. All authors contributed to the article and approved the submitted version.

\section{FUNDING}

Funding for this article was provided by the National Institutes of Health through grants (AG055378, AG062220, AG066518, AG028084, and OD011092) and the state of Arizona, and Arizona Department of Health Services (ADHS14-052688).

pathology in the chimpanzee brain. Philos. Trans. R. Soc. Lond. B. Biol. Sci. 375:20190619. doi: 10.1098/rstb.2019.0619

Egan, M. F., Kost, J., Voss, T., Mukai, Y., Aisen, P. S., Cummings, J. L., et al. (2019). Randomized trial of verubecestat for prodromal Alzheimer's disease. N. Engl. J. Med. 380, 1408-1420. doi: 10.1056/NEJMoa1812840

Emborg, M. E. (2017). Nonhuman primate models of neurodegenerative disorders. J. ILAR 58, 190-201. doi: 10.1093/ilar/ilx021

Farlow, M., Arnold, S. E., Van Dyck, C. H., Aisen, P. S., Snider, B. J., Porsteinsson, A. P., et al. (2012). Safety and biomarker effects of solanezumab in patients with Alzheimer's disease. Alzheimers Dement. 8, 261-271. doi: 10.1016/j.jalz.2011.09. 224

Flurkey, K., Currer, J. M., and Harrison, D. E. (2007). “The mouse in biomedical research," in History, Wild Mice, and Genetics, 2nd Edn, eds J. G. Fox, M. T. Davisson, F. W. Quimby, S. W. Barthold, C. E. Newcomer, A. L. Smith, et al. (Cambridge, MA: Division of Comparative Medicine, MIT), 637-372.

Forny-Germano, L., Lyra e Silva, N. M., Batista, A. F., Brito-Moreira, J., Gralle, M., Boehnke, S. E., et al. (2014). Alzheimer's disease-like pathology induced by amyloid- $\beta$ oligomers in nonhuman primates. J. Neurosci. 34, 13629-13643. doi: 10.1523/JNEUROSCI.1353-14.2014

Freeman, S. H., Kandel, R., Cruz, L., Rozkalne, A., Newell, K., Frosch, M. P., et al. (2008). Preservation of neuronal number despite agerelated cortical brain atrophy in elderly subjects without Alzheimer disease. J. Neuropathol. Exp. Neurol. 67, 1205-1212. doi: 10.1097/NEN.0b013e31818 $\mathrm{fc} 72 \mathrm{f}$

Furcila, D., Defelipe, J., and Alonso-Nanclares, L. (2018). A study of amyloid$\beta$ and phosphotau in plaques and neurons in the hippocampus of Alzheimer's disease patients. J. Alzheimer's Dis. 64, 417-435. doi: 10.3233/JAD180173

Giannakopoulos, P., Silhol, S., Jallageas, V., Mallet, J., Bons, N., Bouras, C., et al. (1997). Quantitative analysis of tau protein-immunoreactive accumulations and beta amyloid protein deposits in the cerebral cortex of the mouse lemur, Microcebus murinus. Acta Neuropathol. 94, 131-139. doi: 10.1007/ s004010050684

Giannaris, E. L., and Rosene, D. L. (2012). A stereological study of the numbers of neurons and glia in the primary visual cortex across the lifespan of male and female rhesus monkeys. J. Comp. Neurol. 520, 3492-3508. doi: 10.1002/cne. 23101

Haass, C., and Levin, J. (2019). Hat die Alzheimer-Forschung versagt?: das scheitern amyloidbasierter klinischer Studien. Der Nervenarzt 90, 884-890. doi: 10.1007/s00115-019-0751-1

Hara, Y., Rapp, P. R., and Morrison, J. H. (2012). Neuronal and morphological bases of cognitive decline in aged rhesus monkeys. Age 34, 1051-1073. doi: 10.1007/s11357-011-9278-5 
Harada, C. N., Natelson Love, M. C., and Triebel, K. L. (2013). Normal cognitive aging. Clin. Geriatr. Med. 29, 737-752. doi: 10.1016/j.cger.2013.07.002

Heilbroner, P. L., and Kemper, T. L. (1990). The cytoarchitectonic distribution of senile plaques in three aged monkeys. Acta Neuropathol. 81, 60-65. doi: 10.1007/BF00662638

Herndon, J. G., Moss, M. B., Rosene, D. L., and Killiany, R. J. (1997). Patterns of cognitive decline in aged rhesus monkeys. Behav. Brain Res. 87, 25-34. doi: 10.1016/s0166-4328(96)02256-5

Ingram, D. K., and De Cabo, R. (2017). Calorie restriction in rodents: caveats to consider. Ageing Res. Rev. 39, 15-28. doi: 10.1016/j.arr.2017. 05.008

LaFerla, F. M., and Green, K. N. (2012). Animal models of Alzheimer's disease. Cold Spring Harb. Perspect. Med. 2, 1031-1085. doi: 10.1016/B978-0-12-809468-6. 00040- 1

Lai, Z. C., Moss, M. B., Killiany, R. J., Rosene, D. L., and Herndon, J. G. (1995). Executive system dysfunction in the aged monkey: spatial and object reversal learning. Neurobiol. Aging 16, 947-954. doi: 10.1016/0197-4580(95)02014-4

Lyra e Silva, N. M., Gonçalves, R. A., Boehnke, S. E., Forny-Germano, L., Munoz, D. P., and De Feliz, F. G. (2019). Understanding the link between insulin resistance and Alzheimer's disease: insights from animal models. Exp. Neurol. 316, 1-11. doi: 10.1016/j.expneurol.2019.03.016

Marner, L., Nyengaard, J. R., Tang, Y., and Pakkenberg, B. (2003). Marked loss of myelinated nerve fibers in the human brain with age. J. Comp. Neurol. 462, 144-152. doi: $10.1002 / \mathrm{cne} .10714$

Mattison, J. A., Colman, R. J., Beasley, T. M., Allison, D. B., Kemnitz, J. W., Roth, G. S., et al. (2017). Caloric restriction improves health and survival of rhesus monkeys. Nat. Commun. 8:14063. doi: 10.1038/ncomms1 4063

Maust, D. T., Solway, E., Langa, K. M., Kullgren, J. T., Kirch, M., Singer, D. C., et al. (2020). Perception of dementia risk and preventive actions among US adults aged 50 to 64 years. JAMA Neurol. 77, 259-262. doi: 10.1001/jamaneurol.2019. 3946

Mehla, J., Gupta, P., Pahuja, M., Diwan, D., and Diksha, D. (2020). Indian medicinal herbs and formulations for Alzheimer's disease, from traditional knowledge to scientific assessment. Brain Sci. 10:964. doi: 10.3390/ brainsci10120964

Messaoudi, I., Estep, R., Robinson, B., and Wong, S. W. (2011). Nonhuman primate models of human immunology. Antioxid. Redox. Signal. 14, 261-273. doi: 10.1089/ars.2010.3241

Miziak, B., Błaszczyk, B., and Czuczwar, S. J. (2021). Some candidate drugs for pharmacotherapy of Alzheimer's Disease. Pharmaceuticals 14:458. doi: 10.3390/ ph14050458

Moore, T. L., Killiany, R. J., Herndon, J. G., Rosene, D. L., and Moss, M. B. (2003). Impairment in abstraction and set shifting in aged rhesus monkeys. Neurobiol. Aging 24, 125-134. doi: 10.1016/s0197-4580(02) 00054-4

Moore, T. L., Killiany, R. J., Herndon, J. G., Rosene, D. L., and Moss, M. B. (2006). Executive system dysfunction occurs as early as middle-age in the rhesus monkey. Neurobiol. Aging 27, 1484-1493. doi: 10.1016/j.neurobiolaging.2005. 08.004

Nagahara, A. H., Bernot, T., and Tuszynski, M. H. (2010). Age-related cognitive deficits in rhesus monkeys mirror human deficits on an automated test battery. Neurobiol. Aging 31, 1020-1031. doi: 10.1016/j.neurobiolaging.2008.07. 0070

Nelson, P. T., Alafuzoff, I., Bigio, E. H., Bouras, C., Braak, H., Cairns, N. J., et al. (2012). Correlation of Alzheimer disease neuropathologic changes with cognitive status: a review of the literature. J. Neuropath. Exp. Neurol. 71, 362-381. doi: 10.1097/NEN.0b013e31825018f7

O'Brien, R. J., and Wong, P. C. (2011). Amyloid precursor protein processing and Alzheimer's disease. Ann. Rev. Neurol. 34, 185-204. doi: 10.1146/annurevneuro-061010-113613

Oikawa, N., Kimura, N., and Yanagisawa, K. (2010). Alzheimer-type tau pathology in advanced aged nonhuman primate brains harboring substantial amyloid deposition. Brain Res. 1315, 137-149. doi: 10.1016/j.brainres.2009. 12.005

Ostrowitzki, S., Lasser, R. A., Dorflinger, E., Scheltens, P., Barkhof, F., Nikolcheva, T., et al. (2017). A phase III randomized trial of gantenerumab in prodromal
Alzheimer's disease. Alzheimers Res. Ther. 9, 1-15. doi: 10.1186/s13195-0170318-y

Paspalas, C. D., Carlyle, B. C., Leslie, S., Preuss, T. M., Crimins, J. L., Huttner, A. J., et al. (2018). The aged rhesus macaque manifests Braak stage III/IV Alzheimer'slike pathology. Alzheimers Dement. 14, 680-691. doi: 10.1016/j.jalz.2017.11. 005

Perez, S. E., Raghanti, M. A., Hof, P. R., Kramer, L., Ikonomovic, M. D., Lacor, P. N., et al. (2013). Alzheimer's disease pathology in the neocortex and hippocampus of the western lowland gorilla (Gorilla gorilla gorilla). J. Comp. Neurol. 521, 4318-4238. doi: 10.1002/cne.23428

Peters, A., Leahu, D., Moss, M. B., and McNally, K. J. (1994). The effects of aging on area 46 of the frontal cortex of the rhesus monkey. Cereb. Cortex 4, 621-635. doi: $10.1093 /$ cercor/4.6.621

Peters, A., Morrison, J. H., Rosene, D. L., and Hyman, B. T. (1998). Feature article: are neurons lost from the primate cerebral cortex during normal aging? Cereb. Cortex 8, 295-300. doi: 10.1093/cercor/8.4.295

Peters, A., Rosene, D. L., Moss, M. B., Kemper, T. L., Abraham, C. R., Tigges, J., et al. (1996). Neurobiological bases of age-related cognitive decline in the rhesus monkey. J. Neuropathol. Exp. Neurol. 55, 861-874. doi: 10.1097/00005072199608000-00001

Rapp, P. (1993). Neuropsychological analysis of learning and memory in the aged nonhuman primate. Neurobiol. Aging 14, 627-629. doi: 10.1016/0197-4580(93) 90050-1

Rapp, P. R., and Amaral, D. G. (1989). Evidence for task-dependent memory dysfunction in the aged monkey. J. Neurosci. 9, 3568-3576. doi: 10.1523/ jneurosci.09-10-03568.1989

Rhesus Macaque Genome Sequencing Analysis Consortium, Gibbs, R. A., Rogers, J., Katze, M. G., (2007). Evolutionary and biomedical insights from the rhesus macaque genome. Science 316, 222-234. doi: 10.1126/science.11 39247

Roberts, D. E., Killiany, R. J., and Rosene, D. L. (2012). Neuron numbers in the hypothalamus of the normal aging rhesus monkey: stability across the adult lifespan and between the sexes. J. Comp. Neurol. 520, 1181-1197. doi: 10.1002/ cne. 22761

Schott, J. M. (2017). The neurology of ageing: what is normal? Pract. Neurol. 17, 172-182. doi: 10.1136/practneurol-2016-001566

Schultz, C., Dehghani, F., Hubbard, G. B., Thal, D. R., Struckhoff, G., Braak, E., et al. (2000). Filamentous tau pathology in nerve cells, astrocytes, and oligodendrocytes of aged baboons. J. Neuropathol. Exp. Neurol. 59, 39-52. doi: 10.1093/jnen/59.1.39

Sevigny, J., Chiao, P., Bussière, T., Weinreb, P. H., Williams, L., Maier, M., et al. (2016). The antibody aducanumab reduces $A \beta$ plaques in Alzheimer's disease. Nature 537, 50-56. doi: 10.1038/nature19323

Sharma, G., Huo, A., Kimura, T., Shiozawa, S., Kobayashi, R., Sahara, N., et al. (2019). Tau isoform expression and phosphorylation in marmoset brains. J. Biol. Chem. 94, 11433-11444. doi: 10.1074/jbc.RA119. 008415

Smith, D. E., Rapp, P. R., McKay, H. M., Roberts, J. A., and Tuszynski, M. H. (2004). Memory impairment in aged primates is associated with focal death of cortical neurons and atrophy of subcortical neurons. J. Neurosci. 24, 4373-4381. doi: 10.1523/JNEUROSCI.4289-03. 2004

Smith, R., Wibom, M., Pawlik, D., Englund, E., and Hansson, O. (2019). Correlation of in vivo [18F] flortaucipir with postmortem alzheimer disease tau pathology. JAMA Neurol. 76, 310-317. doi: 10.1001/jamaneurol.2018.3692

Sorwell, K. G., Garten, J., Renner, L., Weiss, A., Garyfallou, V. T., Kohama, S. G., et al. (2012). Hormone supplementation during aging: how much and when? Rejuvenation Res. 15, 128-131. doi: 10.1089/rej.2011. 1251

Squire, L. R., Genzel, L., Wixted, J. T., and Morris, R. G. (2015). Memory consolidation. Cold Spring Harb. Perspect. Biol. 7:a021766. doi: 10.1101/ cshperspect.a021766

Stonebarger, G. A., Urbanski, H. F., Woltjer, R. L., Vaughan, K. L., Ingram, D. K., Schultz, P. L., et al. (2020). Amyloidosis increase is not attenuated by longterm calorie restriction or related to neuron density in the prefrontal cortex of extremely aged rhesus macaques. GeroScience 42, 1733-1749. doi: 10.1007/ s11357-020-00259-0 
Uno, H. (1993). The incidence of senile plaques and multiple infarction in aged macaque brain. Neurobiol. Aging 14, 673-674. doi: 10.1016/0197-4580(93) 90067-1

U.S. Census Bureau (2020). An Aging World: 2020. Available online at: https://mtgis-portal.geo.census.gov/arcgis/apps/MapSeries/index.html?appid= 3d832796999042daae7982ff36835e2e (accessed June 8, 2021).

U.S. Food \& Drug Administration (2021). FDA Grants Accelerated Approval for Alzheimer's Drug. Available online at: https://www.fda.gov/news-events/pressannouncements/fda-grants-accelerated-approval-alzheimers-drug (accessed June 8, 2021).

Vogel, J. W., Young, A. L., Oxtoby, N. P., Smith, R., Ossenkoppele, R., Strandberg, O. T., et al. (2021). Four distinct trajectories of tau deposition identified in Alzheimer's disease. Nat. Med. 27, 871-881. doi: 10.1038/s41591-021-01309-6

Voytko, M. L. (1999). Impairments in acquisition and reversals of twochoice discriminations by aged rhesus monkeys. Neurobiol. Aging 20, 617-627. doi: 10.1016/s0197-4580(99)00097-4

Webster, S. J., Bachstetter, A. D., Nelson, P. T., Schmitt, F. A., and Van Eldik, L. J. (2014). Using mice to model Alzheimer's dementia: an overview of the clinical disease and the preclinical behavioral changes in 10 mouse models. Front. Genet. 5:1-23. doi: 10.3389/fgene.2014.00088
Zhang, J., Chen, B., Lu, J., Wu, Y., Wang, S., Yao, Z., et al. (2019). Brains of rhesus monkeys display $\mathrm{A} \beta$ deposits and glial pathology while lacking $\mathrm{A} \beta$ dimers and other Alzheimer's pathologies. Aging Cell 18:e12978. doi: 10.1111/acel.12978

Conflict of Interest: The authors declare that the research was conducted in the absence of any commercial or financial relationships that could be construed as a potential conflict of interest.

Publisher's Note: All claims expressed in this article are solely those of the authors and do not necessarily represent those of their affiliated organizations, or those of the publisher, the editors and the reviewers. Any product that may be evaluated in this article, or claim that may be made by its manufacturer, is not guaranteed or endorsed by the publisher.

Copyright (c) 2021 Stonebarger, Bimonte-Nelson and Urbanski. This is an open-access article distributed under the terms of the Creative Commons Attribution License (CC BY). The use, distribution or reproduction in other forums is permitted, provided the original author(s) and the copyright owner(s) are credited and that the original publication in this journal is cited, in accordance with accepted academic practice. No use, distribution or reproduction is permitted which does not comply with these terms. 